\title{
Technique of making fish illustration 16: gyotaku, direct prints from fish specimens
}

Don E. McAllister

Linn-Tarn Prints, 2883 Otterson Drive, Ottawa, Ontario KIV 7B2, Canada

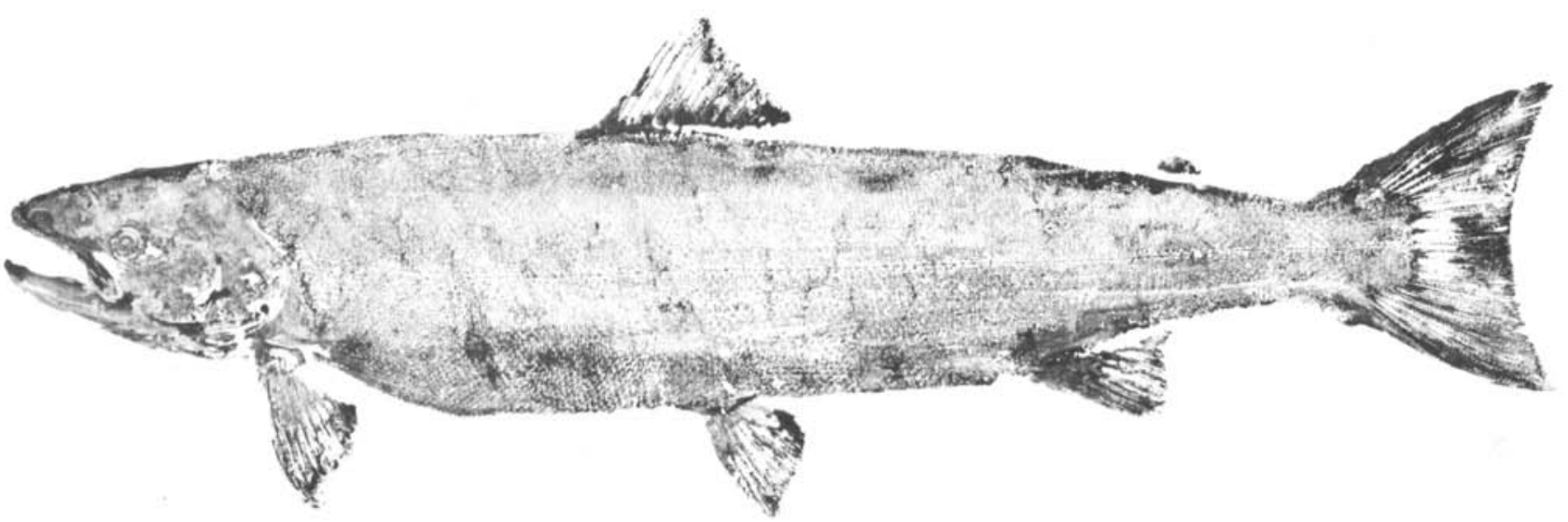

Atlantic salmon, Salmo salar Linnaeus, from Nabisibi River northern Gulf of St. Lawrence, $q, 4$ year river, 2 year sea. Specimen $73 \mathrm{l}$ mm $\left(28.8^{\prime \prime}\right)$ TL., about $3.7 \mathrm{~kg}(8.1 \mathrm{lb})$. Original print by Dr. Geoff Power using direct technique no. 2.

Technique: The illustration was made by the traditional Japanese technique called gyotaku, using the textured surface of the fish itself, ink and paper. The side of the fish to be printed is first washed and dried, then one of three basic methods may be tried: 1) the fish is inked and the inked side is pressed down onto paper, rolled if necessary to print the dorso-lateral surfaces, and the vertical fins are spread and pressed against the paper; 2) the upper side of the fish is inked, thin paper is lowered carefully down onto the fish and wrapped over the inked surface, then removed; or 3) dampened paper is molded over one side of a fish and, when the surface dries, pigment is dabbed on using a wad of cotton or specially made applicator which colors elevated parts. 1) \& 2) are called direct, 3) indirect method.

The paper used should be absorbent and not tear too easily when moist. A variety of inks or paints can be used. Japanese Sumi ink may be replaced by India ink; avoid toxic pigments if the fish is going to be eaten. In applying pigment one may duplicate the patterns or the color of the fish. Avoid shifting the fish or the paper, otherwise a double image may result.

The artist, Dr. Geoff Power, born in England, has been studying salmonids, including the Atlantic salmon, in North America for over 25 years. He learned the method early in his career when looking for a technique to accurately record fish morphology.

The species: The Atlantic salmon was found on both sides of the North Atlantic, from Portugal to the Arctic Circle in the east and from northern Québec to the Connecticut River, as well as Iceland and southern Greenland, as anadromous and sometimes landlocked populations. Over much of its range its populations have been reduced or extirpated by dams, overfishing and pollution - most recently by acid rain.
To: Linn-Tarn Prints

2883 Otterson Drive

Ottawa, Ontario K1V 7B2, CANADA
Prints of the above drawing, $11 \times 17$ inches $(28 \times 43 \mathrm{~cm})$, blue ink on hand-made paper, suitable for framing and gifts may be ordered with this form.

NAME:

PLEASE

ADDRESS: 